\title{
Parasitoides nativos en el control de la polilla del brote del pino (Rhyacionia buoliana): ¿Un complejo funcional?
}

Native parasitoids in pine shoot moth control (Rhyacionia buoliana): a functional complex?

C.D.O.: 453

DOLly M. LANFRANCO, ANGEliCA M. AgUilar, RODRIGO A. HORCOS, JAIME F. BÜCHNER

Instituto de Silvicultura, Universidad Austral de Chile,

Casilla 567, Valdivia, Chile.

\section{SUMMARY}

The control incidence of each of the native parasitoids associated with Rhyacionia buoliana Schiff. is informed. The efficacy of these biocontrol agents and the projection of the generalist-specialist relation in this case of forest importance is commented.

Coccygomimus fuscipes (Brullé) appears as the only species able to complement the parasitism exerted by Orgilus obscurator Ness.

\section{RESUMEN}

Se informa acerca de la incidencia en el control de las especies nativas de parasitoides que se han asociado a Rhyacionia buoliana Schiff. Se comenta sobre la eficacia de estos biocontroladores y de cómo se proyecta la relación generalista-especialista para este caso de importancia en el sector forestal. Coccygomimus fuscipes (Brullé) se perfila como la única especie que podría complementar el control que ejerce Orgilus obscurator Ness.

\section{INTRODUCCION}

Oficialmente la interacción Rhyacionia buolianaPinus radiata se inicia en Chile en 1985 (Cerda et al., 1985). No obstante, se estima que la polilla del brote del pino está en el país desde comienzos de la decada de los 80. Tratándose de dos especies introducidas, esta interacción ha ido generando una historia que está siendo abordada desde diferentes ángulos.

Por otra parte, el desarrollo del sector forestal chileno está sustentado en Pinus radiata D. Don, especie de rápido crecimiento y cuyas plantaciones hoy en día cubren cerca de 1.500 .000 ha. El $70 \%$ de ellas son menores de 10 años y son consideradas como de alta susceptibilidad a Rhyacionia buoliana Schiff. (Lepidoptera: Tortricidae) (Ide et al., 1992; Lanfranco et al., 1992a; 1992b). Esta

Proyecto FONDECYT 0930-91 última ha conquistado gradualmente una mayor y mejor adaptación biológica revelada por algunos indicadores de su dinámica poblacional: nivel de infestación (árboles infestados), grado de infestación (brotes infestados por árbol), densidad poblacional (larvas o pupas vivas por árbol), sobrevivencia (nivel de población que termina su ciclo), área de distribución (desde la X a la VII Región), entre otros (Ide, 1992; .Lanfranco et al., 1992a; Lanfranco, 1992).

Estos antecedentes, sumados a que $P$. radiata es para este agente causal de daño un recurso ilimitado, han permitido que $R$. buoliana haya colonizado, se haya establecido y esté aún en una fase de incremento poblacional en toda su área de distribución actual. Por otra parte, como en la $\mathrm{X}$ Región ya está causando daño económico, ha adquirido la condición de plaga. Resultados recientes indican, sin embargo, que $R$. buoliana en la $\mathrm{X}$ Región está aún lejos de alcanzar la capacidad de carga que el sistema le permite, por lo que es 
esperable que todavía aumenten las poblaciones (Lanfranco et al., 1992a). En el resto de su área de distribución la conducta de $R$. buoliana es, conforme a los antecedentes existentes, la correspondiente a las fases de colonización y establecimiento, con densidades poblacionales menores, pero incrementos significativos entre una generación y otra.

Es así como frente a un escenario distinto al de hoy, en 1989, el sector forestal asumió la opción de controlar biológicamente a $R$. buoliana, para lo cual, en un programa pionero para el sector, se introdujo desde Europa un parasitoide específico: Orgilus obscurator Ness (Hymenoptera: Braconidae). La decisión de introducir esta especie estuvo apoyada por los siguientes atributos de Orgilus: su especificidad, su capacidad para marcar la larva parasitada, su presunta eficiencia sobre $R$. buoliana, sin enemigos naturales y la estrategia koinobionte. Esta última le permite parasitar los primeros estadios de polilla, evitando que cause gran daño al pino. Un eficiente manejo del ciclo de Orgilus permitió sincronizarlo con el de polilla, se le crió masivamente bajo condiciones de laboratorio y comenzó a liberarse en algunos puntos de la X Región como adultos o como implantes de larvas de polillas parasitadas (Cisternas y Villagra, 1991).

Entretanto algunos parasitoides nativos se han ido adaptando al recurso polilla, configurando hoy un complejo de parasitoides que se asocian a su ciclo de vida en etapas posteriores a lo que lo hace $O$. obscurator, en lo que parece ser una repartición del recurso (Porter, 1970; Lanfranco y Cerda, 1986; Lanfranco et al., 1991).

En este trabajo se entregan los resultados de dos años consecutivos de monitoreo de la actividad de los parasitoides nativos, tanto en terreno como en laboratorio, incursiona en algunas reflexiones explicativas acerca de la eficacia de estos biocontroladores y da antecedentes sobre cómo se está dando en Chile y para esta situación la controversia clásica de los biocontroladores generalistas contra los especialistas (Lanfranco, 1992). Estos resultados complementan los publicados por Lanfranco et al., 1991.

\section{MATERIAL Y METODO}

Area de estudio. Las investigaciones se llevaron a cabo principalmente en los predios Peleco (P), Oldemburgo (O) y Huequecura $(\mathrm{H})$, provincia de Valdivia, X Región, abarcando distintas clases de sitio y edades de las plantaciones. Además, duran- te los meses de diciembre y enero (91-93) se efectuó un muestreo intensivo en las siguientes localidades, ubicadas en la misma Provincia y Región: Catamutún (C), La Unión (U), Cruce Los Tambores (CT), Cuesta del Cero (CC), Chaqueán ( $\mathrm{Ch})$, Isla Teja (IT), Huichahue (H), Lipingue (L), Los Lagos (LL), Los Encinos (LE), Los Copihues (LC), Los Ulmos (LU) y Río Bueno (RB) (figura 1).

Método de muestreo. Para la ejecución de los objetivos propuestos para este estudio se emplearon dos técnicas de muestreo. La primera consistió en aislar, cubriendo con bolsas de tul, brotes infestados con larvas de $R$. buoliana, lo cual se hizo quincenalmente desde septiembre de cada año hasta la primera quincena de diciembre, período en que todo el gremio de parasitoides ya está asociado a R. buoliana (Lanfranco et al., 1991). Esta técnica de muestreo permitió tomar estos registros como testigo. Las bolsas se retiraron a fines de enero de los años 92 y 93, momento en que la polilla del brote ha alcanzado el $100 \%$ de emergencia de adultos (Cerda et al., 1986).

La segunda técnica consistió en la extracción de muestras de larvas y/o pupas para crianza en condiciones de laboratorio. La extracción de muestras se realizó quincenalmente de septiembre a la primera quincena de diciembre y semanalmente hasta fines de enero de cada año. La intensificación de muestreo a fines de cada año se debió a que en ese período ocurre la máxima emergencia de $R$. buoliana y de parasitoides. Las dos técnicas,de muestreo se emplearon en los tres predios; sin embargo para la técnica de crianza se utilizaron además otras localidades, en las cuales se efectuó una sola extracción de muestras

Durante los dos años de duración del proyecto, tanto para el trabajo de terreno como de laboratorio, se utilizaron como base 100 muestras por fecha de muestreo y localidad, las que se aislaron y/ o extrajeron en igual proporción de los estratos superior y medio del árbol.

A las muestras en laboratorio se les extrajeron las larvas y/o pupas y cada una fue colocada en una placa plástica (5 $\mathrm{cm}$ de diámetro), donde las larvas tenían como alimento brotes de $P$. radiata y las pupas se colocaron sobre papel filtro. En laboratorio estas muestras fueron desinfectadas con una solución de hipoclorito de sodio al $5 \%$ para brotes y placas plásticas y al $1 \%$ para larvas y pupas. Este proceso se realizó en una cámara de inoculación, y luego las placas se mantuvieron en una cámara climatizada con registros diarios de 


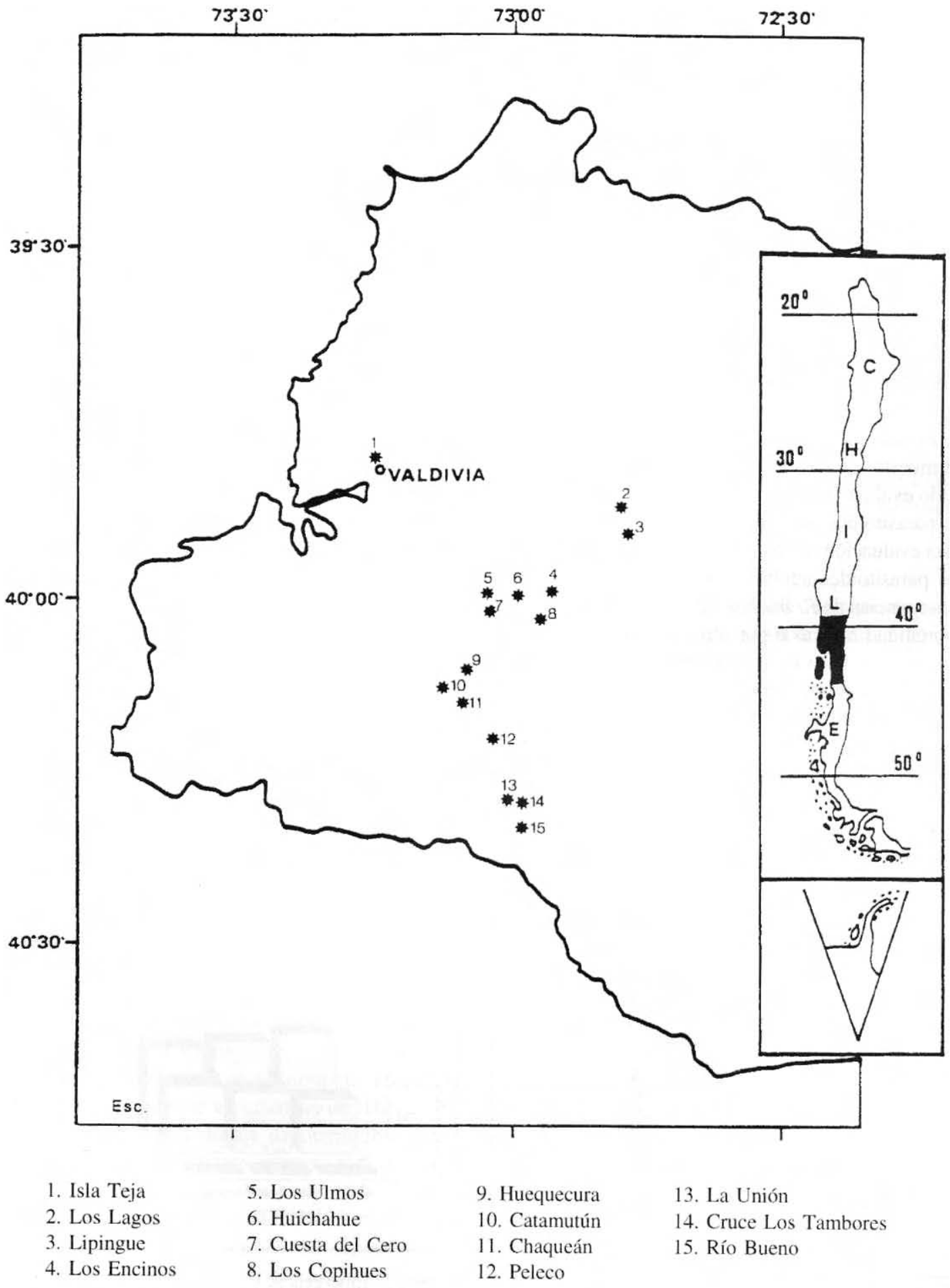

Figura 1. Distribución de puntos de muestreo en la provincia de Valdivia, X Región.

Distribution of the sampling plots at the Valdivia province, $\mathrm{X}$ Region 
temperatura y humedad $\left(20-22^{\circ} \mathrm{C}\right.$ y $50-70 \%$, respectivamente). Tanto la placa como el brote se renovaban cada ocho días, bajo el mismo protocolo de desinfección, manteniéndose en estas condiciones hasta la emergencia de los adultos, ya sea del agente de daño o de los parasitoides.

Identificación de los parasitoides asociados. Tanto de los brotes aislados en terreno como de las crianzas de laboratorio se obtuvieron diversas especies de parasitoides, las que fueron identificadas mediante el apoyo de una colección entomológica de referencia, claves taxonómicas y bibliografía (Porter, 1967, 1970, 1987; Townes, 1969a y b; Lanfranco, 1974, 1980; Lanfranco et al., 1991; Jerez et al., 1977; Zúñiga y Cisternas, 1989).

Evaluación del control. A partir de las dos ténicas de muestreo y los respectivos tamaños muestrales se pudo evaluar la incidencia de las diferentes especies de parasitoides asociadas al control de $R$. buoliana. Esta evaluación se efectuó sólo con la emergencia de los parasitoides adultos, cotejándose luego con las emergencias de $R$. buoliana para cada muestreo y la mortalidad natural o por otros agentes del insecto.

\section{RESULTADOS}

Emergencia y mortalidad: un marco general. Los resultados por predio y técnica de muestreo se entregan en las figuras 2 a 5 .

Durante la temporada 91-92 (figura 2) los porcentajes de emergencia de $R$. buoliana en Peleco

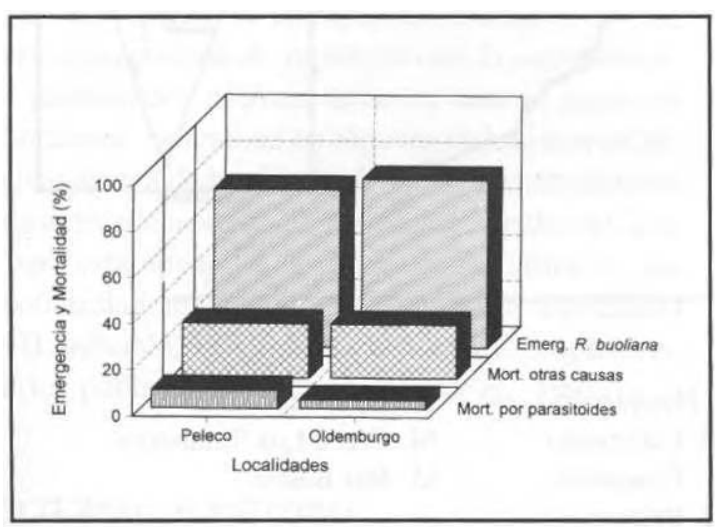

Figura 2. Emergencia y mortalidad (por parasitoides y otras causas) de $R$. buoliana en la técnica de crianza. Peleco $(\mathrm{N}=1123)$ y Oldemburgo $(\mathrm{N}=1187)$. 1991-1992. $R$. buoliana emergency and mortality (by parasitoids and other factors) under rearing. Peleco $(\mathrm{N}=1123)$ and Oldemburgo ( $\mathrm{N}=1187)$. 1991-1992. fueron de un $68.5 \%$ y en Oldemburgo de un $73 \%$. En tanto la mortalidad por parasitoides alcanzó a un $8 \%$ y $4 \%$, respectivamente, no revelando importancia significativa la acción de los biocontroladores. La mortalidad por otras causas (depredadores, hongos, bacterias, deshidratación, resinación), no cuantificadas en forma específica en este ensayo, fue de un $23.5 \%$ en Peleco y de un $23 \%$ en Oldemburgo.

En la temporada 92-93 (figura 3) la mortalidad superó las emergencias en todas las situaciones. Sin embargo, no fueron los parasitoides los responsables de esta mortalidad. En efecto, el hongo Beauveria bassiana y principalmente varias bacterias (en determinación) fueron responsables de estas altas tasas de mortalidad (Peleco 63.7\%, Oldemburgo $58,5 \%$ y Huequecura $54,9 \%$ ). La mortalidad por parasitoides en los mismos predios alcanzó sólo al $1.0 \%, 1.6 \%$ y $1.1 \%$, respectivamente.

Teniendo como base las muestras aisladas secuencialmente en terreno, en los dos predios en estudio durante la temporada 91-92 (figura 4), se observó una alta emergencia de adultos de $R$. buoliana ( $86.3 \%$ en Peleco y $90.5 \%$ en Oldemburgo). Los parasitoides tienen una baja incidencia en el control de $R$. buoliana, alcanzando sólo al $1.2 \%$ y el $0.5 \%$ de control en ambos predios, respectivamente. Lo atribuible a otras causas fue de un $12,5 \%$ en Peleco y un $9 \%$ en Oldemburgo.

La figura 5 muestra lo ocurrido en la técnica de aislamiento de brotes en la temporada 92-93. Se observa que las emergencias de $R$. buoliana superan

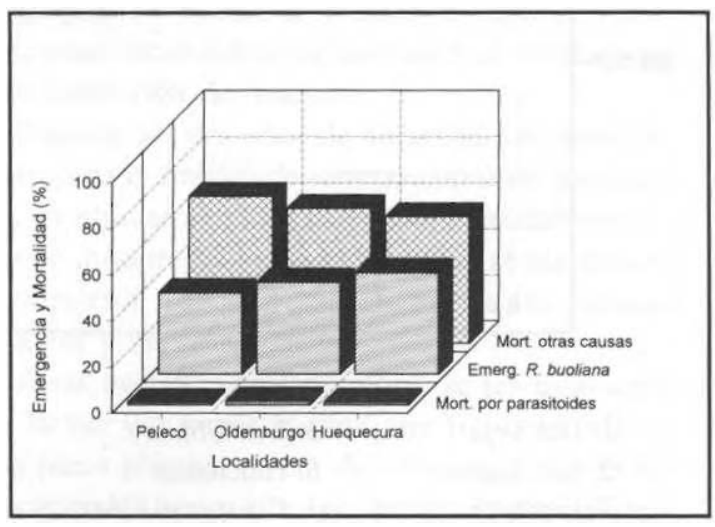

Figura 3. Emergencia y mortalidad (por parasitoides y otras causas) de $R$. buoliana en la técnica de crianza. Peleco (N=1235), Oldemburgo $(\mathrm{N}=749)$ y Huequecura $(\mathrm{N}=714)$. 1992-1993.

$R$. buoliana emergency and mortality (by parasitoids and other factors) under the rearing modality. Peleco $(\mathrm{N}=1235)$, Oldemburgo $(\mathrm{N}=749)$ and Huequecura $(\mathrm{N}=714) .1992-1993$. 


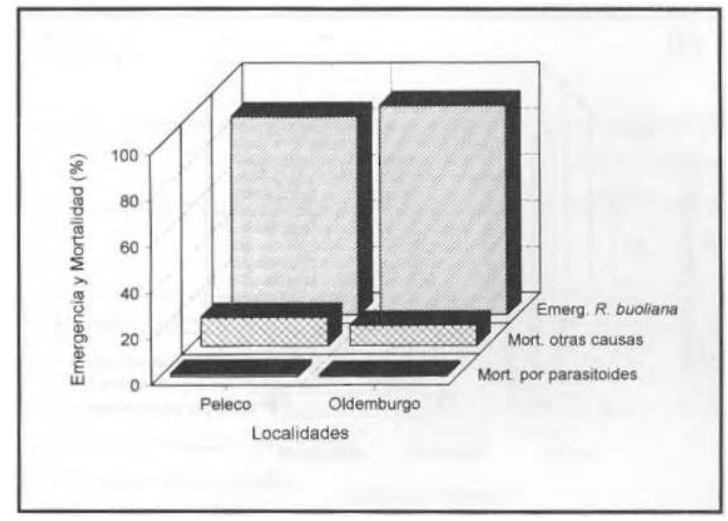

Figura 4. Emergencia y mortalidad de $R$. buoliana (por parasitoides y otras causas) en la técnica de aislamiento de brotes. Peleco $(\mathrm{N}=561)$ y Oldemburgo $(\mathrm{N}=337)$. 19911992.

R. buoliana emergency and mortality (by parasitoids and other factors) under shoot isolation. Peleco $(\mathrm{N}=561)$ and Oldemburgo $(\mathrm{N}=337)$. 1991-1992.

significativamente a la mortalidad en los tres predios, alcanzando un $62.5 \%$ en Peleco, un $56.8 \%$ en Oldemburgo y un $73.4 \%$ en Huequecura. La acción de los biocontroladores fue poco significativa, logrando sólo $0.4 \%, 2.7 \%$ y $2.5 \%$ respectivamente. La mortalidad, provocada por otras causas, en condiciones de terreno, fue mayor que la observada en la primera temporada (91-92) y alcanzó porcentajes de $37.1 \%, 40.5 \%$ y $24.1 \%$, en el mismo orden de los predios señalados anteriormente.

Distribución en el tiempo de la emergencia y la mortalidad. Para expresar el cómo se distribuye la emergencia y la mortalidad a través de los periodos estudiados, se graficaron los porcentajes registrados en cada predio, para cada técnica de muestreo y para cada temporada entre los meses de septiembre y enero (figuras 6A y 6B).

En las figura $6 \mathrm{~A}$ y $6 \mathrm{~B}$ se observa que las emergencias de $R$. buoliana en crianzas de laboratorio se concentran en los meses de noviembre y diciembre, siendo diciembre el mes con mayores registros. Al comparar las dos temporadas se puede observar que en la temporada 91-92 (figura 6A) hubo una emergencia de $R$. buoliana significativamente superior a la temporada 92-93 (figura 6B), incrementándose en ésta la mortalidad por otras causas, la que fue menor en la temporada 91-92. Estas diferencias son atribuibles a la presencia de algunos agentes entomopatógenos, los que bajo las condiciones favorables de crianza en laboratorio se multiplicaron con rapidez.

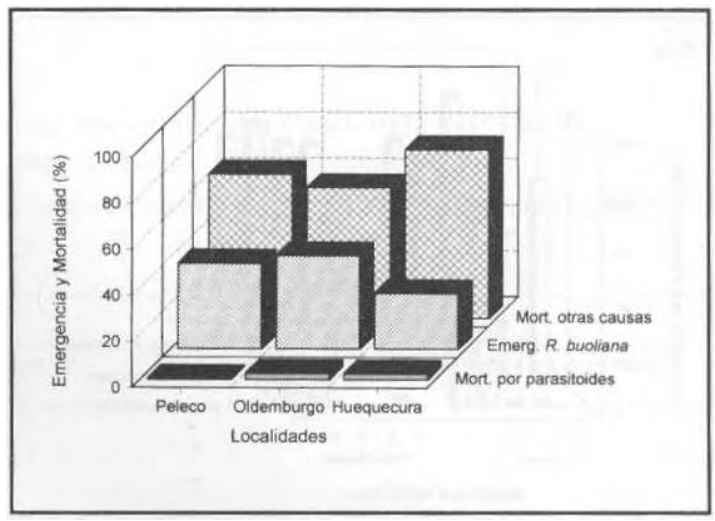

Figura 5. Emergencia y mortalidad de $R$. buoliana (por parasitoides y otras causas) en la técnica de aislamiento de brotes. Peleco $(\mathrm{N}=531)$, Oldemburgo $(\mathrm{N}=294)$, Huequecura $(\mathrm{N}=199)$. 1992-1993.

R. buoliana emergency and mortality (by parasitoids and other factors) under shoot isolation. Peleco $(\mathrm{N}=531)$, Oldemburgo $(\mathrm{N}=294)$ and Huequecura $(\mathrm{N}=199)$. 1992-1993.

La mortalidad por parasitoides, aunque de escasa incidencia, fue levemente superior en la temporada 91-92, donde hubo emergencia de parasitoides preferentemente en diciembre y enero, período en que emergen los adultos. En tanto, en la temporada 92-93 la emergencia ocurrió de un modo similar en los tres predios, pero sólo en diciembre, no registrándose parasitoides en enero. En este estudio ha sido una norma atribuir un grado de control a una especie de parasitoide cuando éste emerge como adulto.

En el caso de los resultados obtenidos a través de la técnica de aislamiento de brotes (figura 7) la tendencia observada con respecto a la emergencia de $R$. buoliana, mortalidad por otras causas y parasitismo son similares a las observadas a través de la técnica de crianza, difiriendo en los meses en que se concentran éstas. Para crianzas abarcó un período de cuatro a cinco meses (figura 6A y 6B), en tanto, en aislamiento de brotes se concentró entre dos y cuatro meses (figura 7A y 7B). Estos antecedentes avalan la idea de que en la última temporada (92-93) hubo una fuerte incidencia de agentes entomopatógenos, principalmente bacterias, las cuales fueron responsables de una mortalidad natural alta, situación que no se había detectado en la temporada anterior (91-92). Es probable que la acción de estas bacterias haya limitado aún más la acción de los parasitoides.

Parasitoides asociados a $R$. buoliana y su contribución al control. Los parasitoides que se de- 



Figura 6. Emergencia y mortalidad de $R$. buoliana durante el período de muestreo. A: Peleco (N=1123) y Oldemburgo (N=1187). 1991-1992. B: Peleco $(\mathrm{N}=1235)$ Oldemburgo $(\mathrm{N}=749)$ y Huequecura $(\mathrm{N}=714)$. 1992- 1993. Crianzas. R. buoliana emergency and mortality during the sampling period. A: Peleco $(\mathrm{N}=1123)$ and Oldemburgo $(\mathrm{N}=1187)$. 1991-1992. B: Peleco ( $\mathrm{N}=235)$, Oldemburgo $(\mathrm{N}=749)$ and Huequecura $(\mathrm{N}=714)$. 1992-1993. Rearings.


Figura 7. Emergencia y mortalidad de R. buoliana durante el período de muestreo. A: Peleco (N=561) y Oldemburgo (N=337). 1991-1992. B: Peleco $(\mathrm{N}=531)$, Oldemburgo $(\mathrm{N}=294)$ y Huequecura $(\mathrm{N}=199)$. 1992-1993. Aislamiento de brotes.

R. buoliana emergency and mortality during the sampling period. A: Peleco ( $\mathrm{N}=561)$ and Oldemburgo $(\mathrm{N}=337)$. 1991-1992. B: Peleco $(\mathrm{N}=531)$, Oldemburgo $(\mathrm{N}=294)$ and Huequecura $(\mathrm{N}=199)$. 1992-1993. Shoot isolation.

tectaron asociados al recurso $R$. buoliana en los distintos predios durante las dos temporadas (9192 y 92-93), obtenidos a través de las técnicas de crianza y de aislamiento de brotes, se muestran en los cuadros 1 y 2 , respectivamente.

Al hacer un análisis del cuadro 1, en condiciones de crianza, se observa que el mayor número de especies de parasitoides se obtuvieron en muestras procedentes de Peleco, donde se detectaron seis especies. En Oldemburgo se obtuvieron sólo cuatro especies de parasitoides. En la temporada 92-93 el número de especies presentes bajó drásticamente, detectándose sólo C. fuscipes (Hymenoptera: Ichneumonidae) en tres predios y O. obscurator en Peleco y Oldemburgo.

A través de la técnica de aislamiento de brotes se obtuvieron dos especies en la temporada 91-92 y tres en la temporada 92-93, siendo las más frecuentes, nuevamente, $C$. fuscipes y $O$. obscurator.

En términos generales, se observa que el parasitoide nativo $C$. fuscipes tiene una presencia en prácticamente todos los predios en ambas temporadas, sin embargo, disminuye el número de especies en el aislamiento de brotes. Por otra parte, $O$. 


\section{CUADRO 1}

Parasitoides asociados a $R$. buoliana detectados en las dos temporadas (1991-1992 y 1992-1993) en los distintos predios. Crianzas.

Associated parasitoids with $R$. buoliana detected in the two seasons (1991-1992 and 1992-1993) in different properties. Rearings.

\begin{tabular}{|c|c|c|c|c|c|}
\hline \multirow[t]{2}{*}{ Parasitoides } & \multicolumn{2}{|c|}{ Temporada 91-92 } & \multicolumn{3}{|c|}{ Temporada 92-93 } \\
\hline & Peleco & Oldemburgo & Peleco & Oldemburgo & Huequecura \\
\hline Coccygomimus fuscipes & + & + & + & + & + \\
\hline Orgilus obscurator & + & + & + & +- & \\
\hline Anacis rubripes & + & + & - & -- & \\
\hline Neocryptopterix hypodyneri & - & + & - & -- & \\
\hline Phorocera casanuevai & + & - & - & -- & \\
\hline Incamya chilensis & + & - & - & -- & \\
\hline
\end{tabular}

+ presencia de parasitoides

- ausencia de parasitoides

\section{CUADRO 2}

Parasitoides asociados a $R$. buoliana detectados en las dos temporadas (91-92 y 92-93) en los distintos predios. Aislamiento de brotes.

Associated parasitoids with $R$. buoliana detected in two seasons (91-92 and 92-93) in different properties. Shoot isolations.

\begin{tabular}{|lcccccc|}
\hline \multirow{2}{*}{ Parasitoides } & \multicolumn{2}{c}{ Temporada 91-92 } & & \multicolumn{2}{c|}{ Temporada 92-93 } \\
\cline { 2 - 3 } \cline { 5 - 6 } & Peleco & Oldemburgo & & Peleco & Oldemburgo Huequecura \\
\hline C. fuscipes & + & + & & + & + & - \\
O. obscurator & - & - & & - & + & + \\
P. casanuevai & + & - & - & - & - \\
I. chilensis & - & - & - & + \\
\hline
\end{tabular}

+ presencia de parasitoides

- ausencia de parasitoides

obscurator como parasitoide introducido también aparece en un importante número de predios, lo que significaría que está en proceso de establecimiento. La presencia de los otros parasitoides nativos es reducida, lo que podría explicarse por lo reciente de su asociación al recurso $R$. buoliana y la inespecificidad respecto de sus hospederos. C. fuscipes, a pesar de ser generalista, ha sido la especie que lleva más tiempo asociada a $R$. buoliana (Lanfranco y Cerda, 1986), por lo tanto, su presencia en casi todos los predios pudiera ser consecuencia de un proceso adaptativo reciente, el cual no lo excluye de su condición de generalista, pero sí puede constituirse en un interesante complemento al control biológico.
Cada una de las especies que se han asociado a $R$. buoliana ejercen un grado de control sobre este recurso. Los registros observados en las crianzas se presentan en las figuras $8 \mathrm{~A}$ y $8 \mathrm{~B}$, en tanto los obtenidos en aislamiento de brotes se entregan en las figuras $9 \mathrm{~A}$ y $9 \mathrm{~B}$.

En la técnica de crianza y en la temporada 91 92 (figura 8A) se observa que aparecen varios agentes de control asociados a la polilla del brote, pero sólo $C$. fuscipes, a pesar de estar presente en prácticamente todos los predios, muestra el porcentaje más alto en Peleco $(8.0 \%)$. En la temporada 92-93 (figura 8B) su contribución es bastante menor, alcanzando un valor global del $2.1 \%$ (Peleco $0,9 \%$, Oldemburgo $0.1 \%$ y Huequecura 

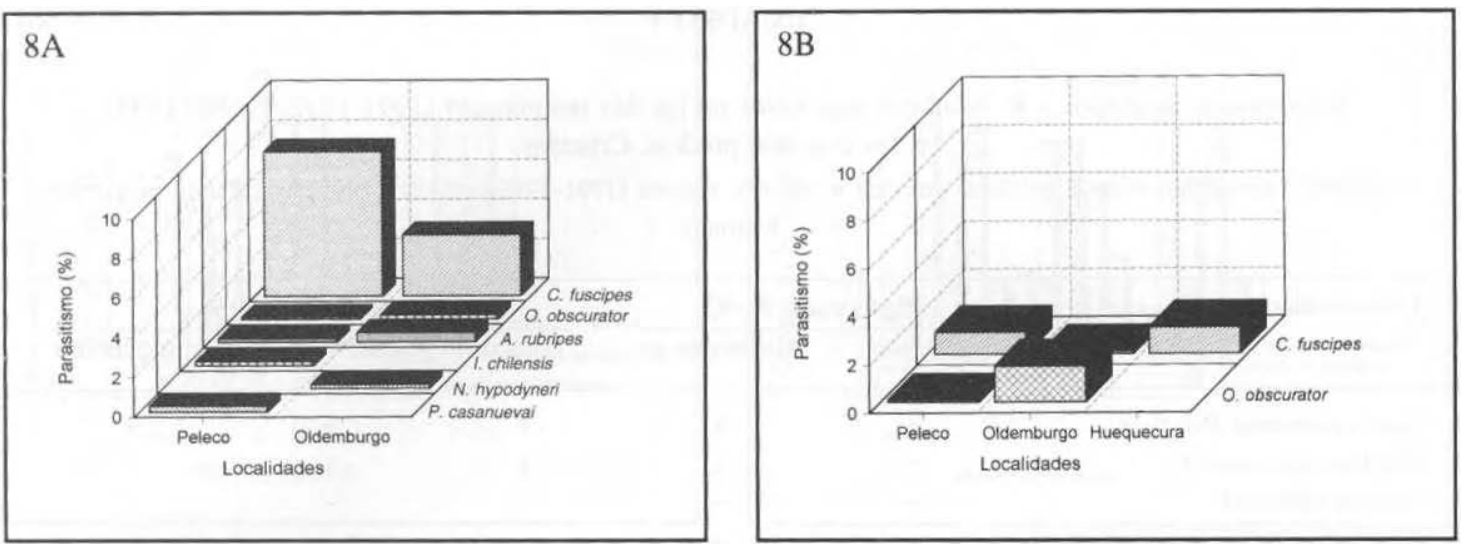

Figura 8. Contribución específica por parasitoides al control de R. buoliana (\%). Crianzas. A: Peleco (N=1123), Oldemburgo (N=1187). 1991-1992. B: Peleco (N=1235), Oldemburgo ( $=749)$, Huequecura $(\mathrm{N}=714)$. $1992-1993$. Specific contribution by parasitoids to $R$. buoliana control (\%). Rearings. A: Peleco (N=1123), Oldemburgo (N=1187). $1991-1992$. B: Peleco ( $\mathrm{N}=1235)$, Oldemburgo ( $\mathrm{N}=749)$, Huequecura $(\mathrm{N}=714)$. 1992-1993.
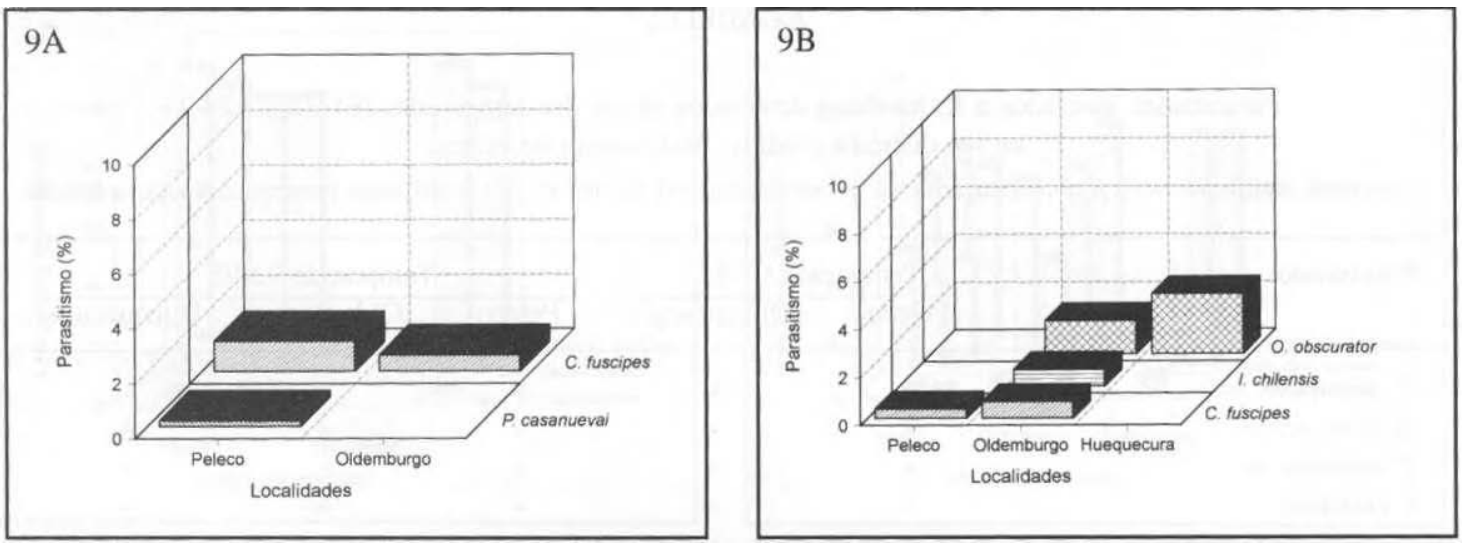

Figura 9. Contribución específica por parasitoides al control de $R$. buoliana (\%). Aislamiento de brotes. A: Peleco ( $\mathrm{N}=561)$, Oldemburgo $(\mathrm{N}=337)$. 1991-1992. B: Peleco $(\mathrm{N}=531)$, Oldemburgo $(\mathrm{N}=294)$, Huequecura $(\mathrm{N}=199)$. 1992-1993.

Specific contribution by parasitoids to $R$. buoliana control (\%). Shoot isolations. A: Peleco ( $\mathrm{N}=561)$, Oldemburgo (N=337). 19911992. B: Peleco ( $N=531)$, Oldemburgo ( $N=294)$, Huequecura $(N=199)$. 1992-1 993.

$1.1 \%)$. O. obscurator, por su parte, aparece en los muéstreos de Peleco y Oldemburgo con un $0.1 \%$ y $1.5 \%$ de control, respectivamente. En este último predio se hicieron liberaciones de $O$. obscurator en 1989. Evaluaciones realizadas por el Instituto Nacional de Investigaciones Agropecuarias (INIA) en 1992 muestran un $20 \%$ de control en este sector. El aporte de las restantes especies de parasitoides es poco significativo.

En el aislamiento de brotes, en la temporada 91-92 (figura 9A), C. fuscipes se detecta en ambos predios, con un nivel de control en Peleco del $1.1 \%$.
Otra especie detectada en este predio fue Phorocera casanuevai (Diptera: Tachinidae), con un aporte de sólo un $0.2 \%$. En la temporada 92-93 (figura 9B) se detectan C. fuscipes, O. obscurator e Incamya chilensis (Diptera: Tachinidae). De estas especies sólo $O$. obscurator muestra un valor de importancia, especialmente en Huequecura y Oldemburgo. Evaluaciones efectuadas por el INIA a inicios del 92 indicaban valores cercanos al $4 \%$ de control en este predio.

Evaluaciones del parasitismo en otras localidades de la X Región. En el período en que $R$. 
buoliana alcanza las fases terminales de su desarrollo (últimos estadios larvales, prepupa y pupa), se colectaron muestras en otras localidades de la región. Los parasitoides emergidos y el porcentaje de control obtenido para el total de la muestra se presentan en las figuras $10 \mathrm{~A}$ y $10 \mathrm{~B}$.

En la temporada 91-92 (figura 10A) se detectaron sólo tres especies de parasitoides: C. fuscipes, $N$. hypodyneri y Anacis rubripes (Hymenoptera: Ichneumonidae), siendo $C$. fuscipes la única relevante, ya que se le encontró en las 10 localidades muestreadas, aunque con notables diferencias en los porcentajes de control en los distintos puntos, los que fluctuaron entre un $1 \%$ (La Unión, LU) y un $69.2 \%$ (Los Copihues, LC). En la temporada 92-93 (figura 10B) las especies obtenidas fueron $C$. fuscipes, $O$. obscurator y $P$. casanuevai. De este grupo $C$. fuscipes fue nuevamente la especie que se presentó en el mayor número de localidades (seis). Los valores fluctuaron entre un $0.4 \%$ (Catamutún, C) y un 56,4\% (Los Ulmos, LU2). Además, los porcentajes de parasitismo cuantificados fueron menores que los obtenidos en la temporada 1991-1992. O. obscurator se encontró sólo en dos predios y con una incidencia muy baja, $1.3 \%$ en Chaqueán ( $\mathrm{Ch}$ ) y $7.7 \%$ en Río Bueno (RB). En tanto, $P$. casanuevai apareció en un solo predio y con una baja incidencia $(0.7 \%)$

\section{DISCUSION}

Aspectos generales. La experiencia de Chile en el manejo de plagas forestales es incipiente. Sin embargo, desde la detección de $R$. buoliana en el

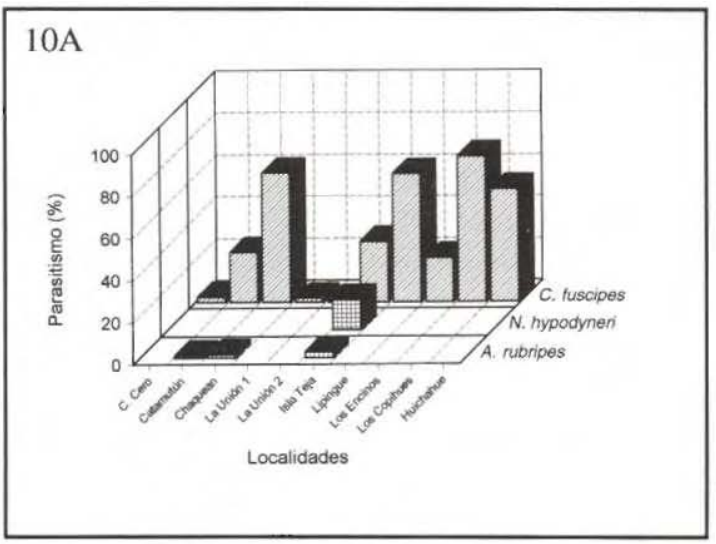

país (Cerda et al., 1985), el sector tomó conciencia del impacto que podría tener la presencia de este insecto asociado al principal recurso forestal, $P$. radiata. En este contexto, optar por el control biológico para el manejo de este insecto-plaga ha sido una estrategia que está acorde con los programas actuales de control biológico que se aplican a nivel mundial, ya que por más de 100 años numerosas plagas de artrópodos se han controlado a través de la introducción de enemigos naturales (Caltagirone, 1981; Hopper et al., 1993).

Conjuntamente con el establecimiento y dispersión de $R$. buoliana en el país, diversas especies de parasitoides nativos, con una conducta oportunista y producto de un proceso adaptativo reciente, se han ido asociando al recurso polilla del brote. Las distintas especies detectadas son generalistas y poseen una amplia distribución geográfica que abarca desde la IV a XII Región, teniendo como hospederos diversas especies de la entomofauna nativa chilena (Cortés e Hichins, 1969; Lanfranco, 1980; Prado, 1991). En consecuencia, la presencia de estos parasitoides sobrepasa el rango de distribución del $P$. radiata en Chile y por ende la actual dispersión que ha alcanzado $R$. buoliana (X a VII Región), constituyéndose en un potencial complemento a $O$. obscurator.

Los resultados obtenidos en este estudio son en gran medida congruentes con los antecedentes obtenidos en otras investigaciones realizadas preliminarmente sobre el tema. Se ha observado que de las especies nativas asociadas al recurso polilla, sólo $C$. fuscipes tiene un valor relativo de importancia, ya que está presente en prácticamente todas las localidades monitoreadas, aunque con ba-

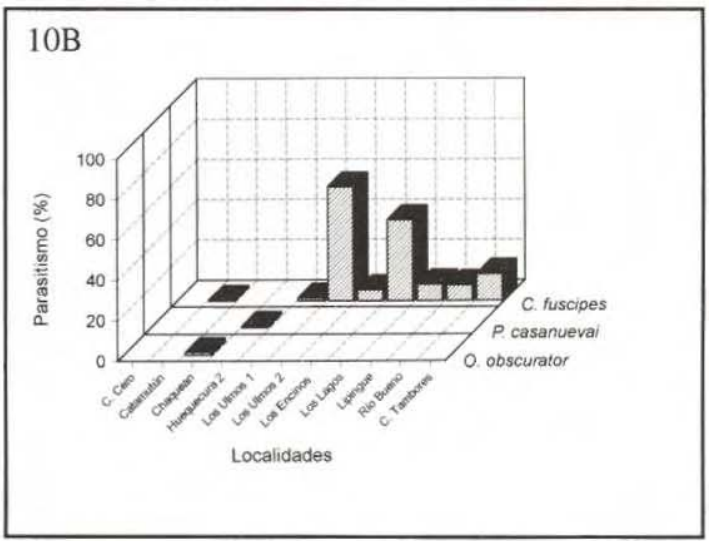

Figura 10. Parasitismo por especie (\%) en otras localidades de la X Región. A: 1991-1992. B: 1992-1993. Parasitism (\%) in others localities of the X Región. A: 1991-1992. B: 1992-1993. 
jos niveles de control, constituyéndose en una especie potencial que a futuro podría complementar la acción de $O$. obscurator. Las restantes especies de parasitoides aparecen muy esporádicamente y con baja incidencia en el control. Es probable que una interacción parasitoide-plaga más eficiente sea sólo cuestión de tiempo (Lanfranco et al., 1991; Büchner, 1993; Horcos, 1993) o, en su efecto, será necesario desarrollar alguna estrategia de manejo del nuevo hábitat que el gremio de parasitoides ha comenzado a ocupar (Syme, 1975; Lewis et al., 1990).

Emergencia y mortalidad con relación a las técnicas de muestreo utilizadas. El emplear dos técnicas de muestreo en este ensayo (crianzas en condiciones de laboratorio y aislamiento de brotes en condiciones de campo) permitió tener una aproximación de lo que ocurre en una parte del ciclo anual de $R$. buoliana en condiciones naturales, verificándose los niveles de emergencia del insecto, la incidencia de los parasitoides como agentes de control y el impacto que puede tener la mortalidad natural, por agentes no siempre predecibles. Estos antecedentes pudieron contrastarse con la información generada en crianzas de laboratorio.

En condiciones de laboratorio (figuras 2 y 3 ) se observó que en la primera temporada del ensayo (91-92) la emergencia de $R$. buoliana superó significativamente a la mortalidad por parasitoides y otras causas. Sin embargo, en la temporada siguiente (92-93), esta situación se revirtió parcialmente, ya que la mortalidad por otras causas, principalmente organismos bacterianos, superó a la emergencia de $R$. buoliana, bajando además significativamente los niveles de parasitismo, los cuales no sobrepasaron en promedio el $1.2 \%$.

Al contrastar la situación anterior con los resultados obtenidos en la técnica de aislamiento de brotes en condiciones de campo, se observó que la emergencia del insecto fue superior en ambas temporadas a la mortalidad por parasitoides y otras causas (figuras 4 y 5). Sin embargo, es necesario destacar que en la temporada 92-93 también se observó un incremento importante en la mortalidad por otras causas, situación que además se observó en condiciones de crianza. Esto permite confirmar que en esta temporada hubo una alta incidencia en la mortalidad natural por agentes entomopatógenos, los cuales, como es obvio, se multiplicaron significativamente en laboratorio, donde la temperatura y humedad eran óptimas. La situación ocurrida es un factor que afecta el comportamiento alimenticio de los parasitoides, ya que su estado fisiológico general se ve alterado con relación a otras necesidades y condiciones, reduciendo con esto su sobrevivencia, búsqueda de alimento y capacidad copulatoria, entre otros (Waage y Hassell, 1982; Lewis et al., 1990).

Con respecto al porcentaje de control logrado por los parasitoides, en general fue bajo en ambas técnicas en las dos temporadas, teniendo la mayor incidencia en la temporada 91-92, en que alcanzó en promedio al $8 \%$ (figura 2). En la segunda temporada, producto de la mortalidad natural ya explicada, ésta bajó significativamente. En la técnica de aislamiento de brotes no hay diferencias de importancia, aun cuando los porcentajes de control alcanzados por los parasitoides no constituyen un gran aporte.

A través de ambas técnicas se pudo detectar, aparte de los parasitoides, otros factores importantes de mortalidad que pueden afectar la población de $R$. buoliana, los cuales también son agentes de control biológico que pudieran, en algún momento, contribuir a la acción de $O$. obscurator $y$ parasitoides nativos. En este estudio no se planteó una cuantificación particular para el caso de enfermedades, por tal razón se han agrupado como un todo (otras causas).

Rol y significancia de los parasitoides asociados a R. buoliana. En términos generales, se asume que un parasitoide eficiente debe tener sincronía total o parcial con el ciclo de su hospedero. En esta interacción parasitoide-hospedero, ambos deberían tender a presentar biologías similares (tiempo generacional, tasa reproductiva y capacidad de dispersión), vale decir, capacidades evolutivas iguales (Ehler, 1990). Esto es válido para koinobiontes como $O$. obscurator $y$ parcialmente válido para idiobiontes como $C$. fuscipes.

Al analizar el rol y significancia que los parasitoides nativos tienen como biocontroladores de $R$. buoliana, se observa que sus niveles de control no son importantes. Sólo $C$. fuscipes puede tener una proyección interesante, ya que está casi en la totalidad de las localidades muestreadas de la X Región. La plasticidad que posee esta especie al utilizar una variedad de hospederos hace factible su incorporación a las plantaciones de pino, que, aunque no es su hábitat natural, ha comenzado a usar recientemente la polilla del brote como recurso alternativo. Esta estrategia de comportamiento se ha definido como un accionar oportu- 
nista. Para mejorar su efectividad en el control biológico, es necesario desarrollar estrategias de manejo del hábitat en el cual se desea que se establezcan, ya que el éxito de los parasitoides depende en gran parte de la disponibilidad de una fuente de alimento adecuada. El establecimiento de plantas con flores en plantaciones de pino es una estrategia que hoy en día, en programas de control biológico, se considera en el manejo forestal (Syme, 1975; Lewis et al., 1990), sin embargo, no ha sido implementada aún en Chile.

La especie $O$. obscurator como parasitoide específico de $R$. buoliana en Europa, y en consecuencia con un historial de coadaptaciones que lo condujeron a esta alta especificidad, ha sido introducido en diversas localidades en plantaciones infestadas con $R$. buoliana, que abarcan desde la X a VII Región (INIA, 1992). En efecto, en este estudio esta especie no representa un elemento clave en el control, mostrando gran variabilidad entre localidades.

Además, debe considerarse que en el hemisferio norte $O$. obscurator y $R$. buoliana son especies nativas. En Chile, hemisferio sur, significó alterar su ciclo para hacerlo compatible a $R$. buoliana donde las plantaciones de pino, el parasitoide y el agente de daño son introducidos. Esto ha significado un proceso de adaptaciones importante en que están involucradas principalmente las condiciones edafoclimáticas (Hopper et al., 1993).

Es posible que el logro de mejores niveles de control con $O$. obscurator sea cuestión de tiempo, ya que la especie debe adaptarse primero a condiciones climático-ambientales y a densidades variables de la plaga, la cual aún está en muchos sectores, en etapa de dispersión y establecimiento. Además, la dispersión es menor especialmente cuando $O$. obscurator debe enfrentar altas densidades de polilla. También es importante conocer cuáles son sus requerimientos de alimento del adulto, ya que según estudios realizados $O$. obscurator se alimenta preferentemente del polen de flores de la familia Umbelliferae (Leius, 1960; Syme, 1975).

Respecto a su acción, el parasitoide introducido reduce el daño físico del brote, parásita en el estadio $2^{\circ}$ a $3^{\circ}$ y completa su desarrollo cuando la polilla está próxima a pupar. Esto ha significado una menor agresividad de $R$. buoliana frente al recurso pino. Situación similar ocurre con $A$. rubripes, pero aún está en etapa incipiente de adaptación a este nuevo hospedero. Otros como $N$. hypodyneri, I. chilensis y $P$. casanuevai parasitan larvas de estadios más avanzados en posdormancia. Por su parte C. fuscipes se asocia en su segunda generación anual en la etapa de prepupa y pupa de polilla, cuando el insecto ya ha hecho prácticamente daño, cumpliendo un ciclo corto para emerger entre diciembre y enero. De momento, es la única especie que se asocia al final del ciclo de polilla, discriminando y seleccionando a su hospedero adecuadamente. A pesar de esto, tienen una gran plasticidad alimenticia, que sumado a que polilla se distribuye espacialmente en forma agregada podría constituirse en un eficiente biocontrolador. De momento, sólo es un complemento a Orgilus $y$ en algunas situaciones puntuales muestra más eficiencia que éste.

Respecto al muestreo realizado en otras localidades de la X Región en las dos temporadas, en los últimos estadios larvales, prepupa y pupa, los resultados obtenidos permiten señalar que el número de especies de parasitoides obtenidos fue sólo de tres especies en cada temporada. Sin embargo, se observó una significativa fluctuación en los niveles de control logrados. La especie $C$. fuscipes aparece nuevamente con la mayor proyección, cuyos niveles de control fluctuaron entre localidades entre un $1 \%$ y un $69 \%(91-92)$ y entre un $0.4 \%$ y un $56 \%$ (92-93). O. obscurator se encontró sólo en dos predios, pero con una baja incidencia.

Los antecedentes obtenidos en los predios de Peleco, Oldemburgo, Huequecura y en otras localidades muestran que no hay una proyección temporal de las otras especies, a excepción de $C$. fuscipes, por lo tanto, no se puede hablar de un complejo de especies que inciden en el control de $R$. buoliana. Es probable que en un futuro próximo, por un proceso adaptativo creciente, la situación actual pudiera revertirse.

\section{BIBLIOGRAFIA}

BÜCHNER, J. 1993. Parasitoides nativos en el control de la polilla del brote del pino (Rhyacionia buoliana): Avances en la identificación y sus estrategias de desarrollo. Tesis Ing. Forestal. Facultad de Ciencias Forestales, Universidad Austral de Chile, Valdivia, Chile, $50 \mathrm{pp}$.

CALTAGIRONE, L.E. 1981. "Landmark examples in classical biological control", Annu. Rev. Entomol. 26:213-232.

CERDA, L., C. JANA-SAENZ, M. BEECHE. 1985. "Detección de la 'Polilla del Brote' en Chile: Rhyacionia buoliana (Schiffermuller, 1876) (Lepidoptera: Tortricidae)", Bol. Soc. Biol. Concepción, Chile 56:161-162.

CERDA, L., C. JANA-SAENZ, O. PUENTES. 1986. "Ciclo de vida en Chile de Rhyacionia buoliana (Schiff.) (Lepidoptera: Tortricidae)", Bol. Soc. Biol. Concepción, Chile 57:201-203. 
CHILE. INSTITUTO NACIONAL DE INVESTIGACIONES AGROPECUARIAS (INIA). 1992. Resultados control biológico. Polilla del brote del pino. Serie Remehue $\mathrm{N}^{\circ} 32$, $4 \mathrm{pp}$.

CISTERnAS E., M. VILlagra. 1991. Polilla del Brote del Pino: Caraterización morfológica, ciclo, daños y control biológico en Chile. Instituto de Investigaciones Agropecuarias. Estación Experimental Remehue. Boletín Técnico $\mathrm{N}^{\circ} 180$, Chile, 19 pp.

EHLER, L. 1990. Introduction strategies in biological control of insect. En: Criticai issues in biological control. $\mathrm{M}$. MACKAUER, L. EHLER and J. ROLAND (eds.). Intercept., London, pp: 111-134.

CORTES, R., N. HICHINS. 1969. Distribución geográfica y huéspedes conocidos de los Taquínidos de Chile (Diptera: Tachinidae). Edit. Univ. de Chile: 5-100

HOPPER, K., R. ROUSH, W. POWELL. 1993. "Management of Genetics of Biological-Control Introductions", Annu. Rev. Entomol. 38:27-51.

HORCOS, R. 1993. Influencia de los parasitoides nativos sobre Rhyacionia buoliana (Schiff.) en la provincia de Valdivia, Décima Región. Tesis Ing. Forestal, Facultad de Ciencias Forestales, Universidad Austral de Chile, Valdivia, Chile, $55 \mathrm{pp}$.

IDE S. 1992. Dinámica poblacional de Rhyacionia buoliana (Schiff.) en plantaciones de Pinus radiata en la Décima Región. Tesis Ing. Forestal, Facultad de Ciencias Forestales, Universidad Austral de Chile, 65 pp.

IDE, S., D. LANFRANCO, R. VALLEJOS, A. AGUILAR. 1992. Algunos aspectos de la dinámica poblacional de Rhyacionia buoliana en una perspectiva histórica. En: Simposio Pinus radiata en Chile. Silvicultura, Manejo y Tecnología, 27 al 30 octubre. Valdivia, Chile. 184-191.

JEREZ, V., D. LANFRANCO, B. ANDRADE. 1977. "Aspectos ecológicos de los Ichneumónidos del Bosque de Quintero", Anales del Museo de Historia Natural. Chile. 10:161168.

LANFRANCO, D. 1974. "Contribución al conocimiento de la Ichneumonofauna de la región de Magallanes. (Hymenoptera: IChneumonidae)", Ans. Inst. Pat. Punta Arenas (Chile), 51 (1 y 2):199-208.

1980. "Contribución al conocimiento de los Ichneumónidos de Chile (Hymenoptera-Ichneumonidae)", Rev. Chilena de Ent. 10:77-84.

1992. Control biológico de la polilla del brote del pino por parasitoides: puntos críticos. En: XIV Congreso Nacional de Entomología. Santiago, Chile. 11-13 noviembre, pp. 51.

LANFRANCO, D., L. CERDA. 1986. "Coccygomimusfuscipes (Hym.: Ichneumonidae): un parasitoide nativo de la polilla del brote, Rhyacionia buoliana (Lep.: Tortricidae). Chile", Bosque 7(1): 36-37.

LANFRANCO, D., J. BÜCHNER, A. AGUILAR, R. HORCOS. 1991. "Parasitoides nativos en el control de la polilla del brote del pino (Rhyacionia buoliana): avances en la identificación del complejo y sus estrategias de desarrollo. Chile", Bosque 12 (1): 69-74.

LANFRANCO D., A. AGUILAR, S. IDE, R. VALLEJOS. 1992a. Algunos aspectos de la dinámica poblacional de Rhyacionia buoliana en una perspectiva histórica. En: Simposio Pinus radiata en Chile. Silvicultura, Manejo y Tecnología. 27 al 30 octubre, Valdivia, Chile, 176-183. 1992b. Evaluación del daño causado por infestación natural de Rhyacionia buoliana sobre plantaciones jóvenes de Pinus radiata en la provincia de Valdivia. Informe de Convenio $\mathrm{N}^{\circ}$ 200. Serie Técnica. Fac. Ciencias Forestales, Universidad Austral de Chile, Valdivia, 48 pp.

LEIUS, K. 1960. "Attractiveness of different foods and flowers to the adults of some hymenopterous parasites", Can. Entomol. 92:369-376.

LEWIS, W., L. VET, J. TUMLINSON, J. VAN LENTERER, D. PAPAJ. 1990. "Variations in parasitoid foraging behavior. Essential element of a sound biological control theory", Environ. Entomol. 19:1183-1193.

PORTER, Ch. 1967. South American Trachysphyrus. Mem. Amer. Ent. Inst. 10, 368 pp. 1970. "A revision of the South American species of Coccygomimus (Hym: Ichnemonidae)", Studia Entom. 13(14): $1-92$.

1987. A revision of the Chilean Mesostenini (Hymenoptera: Ichneumonidae). Contrib. Amer. Ent. Inst. 23(3), $164 \mathrm{pp}$.

PRADO, E. 1991. Artrópodos y sus enemigos naturales asociados a plantas cultivadas en Chile. INIA. Serie Boletín Técnico $\mathrm{N}^{\circ} 169$, Santiago, Chile, 203 pp.

SYME, P.D. 1975. "The effects of flowers on the longevity and fecundity of two native parasites of the European pine shoot moth in Ontario". Environ. Entomol. 4:337-346.

TOWNES, H. 1969a. Genera of Ichneumonidae. Pail. I. Mem. Amer, Ent. Inst. 11, 300 pp.

1969b. Genera of Ichneumonidae. Part. II. Mem. Amer. Ent. Inst. 12, $537 \mathrm{pp}$.

WAAGE, J., M. HASSELL. 1982. "Parasitoids as biological control agents- a fundamental approach", Parasitology 84:241-268.

ZUÑIGA, E., E. CISTERNAS. 1989. Fundamentos, avances, logros y mecanismos para afianzar el impacto biorregulador de $R$. buoliana en Chile. Reunión IICA-SAG. Santiago, Chile, 1-14. 